\title{
Power and energy-scaling of cladding-pumped Raman fibre lasers sources
}

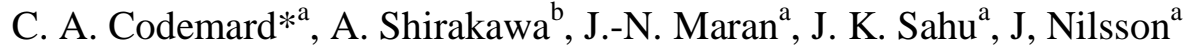 \\ aptoelectronics Research Centre, University of Southampton, Southampton, SO17 1BJ, UK; \\ ${ }^{\mathrm{b}}$ Institute for Laser Science, University of Electro-Communications, Tokyo, Japan
}

\begin{abstract}
We present results on a high-power, high-energy cladding-pumped fibre Raman laser. We first discuss fibre requirements and give a design rule. Then, experimentally, a pulsed cladding-pumped Raman fibre laser is demonstrated with up to $210 \mu \mathrm{J}$ high-brightness pulses. The fibre delivers pulses at the Stokes wavelength of $1112 \mathrm{~nm}$, of $500 \mathrm{~ns}$ duration and $420 \mathrm{~W}$ peak power, with $\mathrm{M}^{2}=1.8$. The linewidth is $6.5 \mathrm{~nm}$ without any wavelength selection. Our result is the highest reported energy and peak power from any fibre Raman source and illustrates the power scalability capability of such devices.
\end{abstract}

Keywords: Cladding-pumped fibre, Raman laser and amplifier.

\section{INTRODUCTION}

Fibre lasers with high-power, high brightness output are desirable for many industrial applications, for example, cutting, marking, and engraving. The current approach relies on cladding-pumped rare-earth doped (mainly Yb-doped) fibre to generate continuous-wave power in excess of $5 \mathrm{~kW}$ [1] with near-diffraction limited beam and high-energy pulses up to $10 \mathrm{~mJ}[1,2]$. For pulsed fibre laser, higher peak power and output energy are obtained with large-core fibre because of the higher energy storage and higher damage threshold those fibre offers. As there are some practical limits on the numerical aperture and core size of single mode fibre, multimode cores, e.g., [2], are also used.

Recently, we and others proposed [3, 4] and demonstrated a new kind of brightness enhancer in continuous-wave [4, 5] and pulsed regimes $[3,6-8]$ using an intensity-dependent nonlinear effect with multimode pumping, namely stimulated Raman scattering (SRS). This is an alternative to conventional rare-earth based fibre lasers. In our case, we claddingpump a double-clad Raman fibre (DCRF) to convert a multimode pump beam into a near diffraction limited output beam at the Stokes wavelength. This is also known as "beam clean-up" [9]. In case of the DCRF, the fibre concept is similar to that of a double-clad rare-earth doped fibre. The Stokes wavelength propagates in a single-mode or few-modes core which facilitates preferential selection of the lower order modes. The pump distribution creates a Raman gain distribution across the core and cladding. Mode-selective feedback can then be used to select the fundamental mode. In addition, the pump distribution is normally such that the gain is highest in the centre of the fibre [10]. Furthermore, the core can be doped with a higher concentration of germanium, which increases the Raman gain of the core. It is also important that all the pump modes overlap with the fundamental mode in the core, to enable transfer of power.

The nearly instantaneous nature of stimulated Raman scattering makes such devices very attractive for single-stage highgain amplification of pulses [6, 7]. Indeed, the Raman gain exists only during the pump pulse, which thus forms a time gate. This negates effects of amplified spontaneous emission which occurs in rare-earth doped fibre devices and limits their energy storage and gain scalability. Thus, pulsed cladding-pumping of Raman fibre laser sources are interesting to directly convert high-energy multimode pulses into diffraction limited ones.

Furthermore, this approach has all the benefits of fibre Raman laser. In particular, it is wavelength-flexible, with the operating wavelength determined by the pump wavelength and limited only by the background loss of the fibre. Importantly, fibre Raman sources have low quantum defect and high slope efficiency. Also, compared to rare-earth doped fibre with higher background loss, longer fibres are typically used, which reduces thermal effects. Finally, these fibres are not known to photodarken in the infrared region.

*cac@orc.soton.ac.uk; phone +44 (0) 238059 2086; www.orc.soton.ac.uk 
Therefore, the output beam quality of any multimode laser source can be improved through SRS in a section of "passive" multimode fibre through the Raman scattering process as a simple add-on. In addition, this cheap and simple solution allows the spectral, spatial and polarization combination of several pump sources into a single Raman fibre laser [11] for further power scaling.

Thus we are exploring the potential of cladding-pump Raman fibre converters for brightness enhancement of multimode high energy pulses. Here we report the highest energy and peak power from any Raman source.

\section{FIBRE DESIGN}

We are mainly interested in the operation at a single Stokes order, in our case the $1^{\text {st }}$ Stokes, because most applications need a specific wavelength and not just power. Also, conversion to higher Stokes orders means longer fibre than that required for $1^{\text {st }}$ Stokes generation which implies additional background loss and higher quantum defect.

In a double-clad fibre, the gain at of the $2^{\text {nd }}$ Stokes, $G_{S R S}^{s 2}$ is given by [12]:

$$
G_{S R S}^{s 2}=g_{R} \frac{L_{e f f}}{A_{e f f}^{s 2, s 1}} P_{s 1} \approx g_{R} \frac{L_{e f f}}{A_{c o r e}} P_{s 1}
$$

where $P_{s 1}$ is the power of the $1^{\text {st }}$ Stokes, $g_{R}$ is the material Raman gain coefficient and $L_{e f f}$ is the device effective length. The effective area $A_{e f f}^{s 2, s 1}$ which corresponds to the interaction region of the $1^{\text {st }}$ and $2^{\text {nd }}$ Stokes, can be approximated here to the core area, $A_{\text {core }}$.

In addition, the pump absorption coefficient into the $1^{\text {st }}$ Stokes, $\alpha_{p}$ is given by:

$$
\alpha_{p}=\frac{\omega_{p}}{\omega_{s 1}} g_{R} \frac{L_{e f f}}{A_{e f f}^{p, s 1}} P_{s 1} \approx \frac{\lambda_{s 1}}{\lambda_{p}} g_{R} \frac{L_{e f f}}{A_{\text {clad }}} P_{s 1}
$$

where $\omega_{p}$ and $\omega_{s}$ are the pump and $1^{\text {st }}$ Stokes optical frequencies with $\lambda_{p}$ and $\lambda_{s}$ their respective wavelength. The effective $A_{e f f}^{p, s 1}$ for the pump and $1^{\text {st }}$ Stokes can also be approximated by the cladding area, $A_{\text {clad }}$.

In a real laser, the $2^{\text {nd }}$ Stokes will becomes important once its gain reach about 16 Nepers $(\sim 70 \mathrm{~dB})$ since it builds from noise. Also, in order to have an efficient power/energy conversion into the $1^{\text {st }}$ Stokes, the pump absorption should be in the region of 2 Nepers $(\sim 8 \mathrm{~dB})$. Thus, the ratio of the gain and pump absorption is:

$$
\frac{G_{S R S}^{s 2}}{\alpha_{p}}<8
$$

Using Eq. (1) and (2) to replace $G_{S R S}^{s 2}$ and $\alpha_{p}$ in Eq. (3), we obtain the following condition:

$$
\frac{A_{\text {clad }}}{A_{\text {core }}}\left(\frac{\lambda_{p}}{\lambda_{s}}\right)=\left(\frac{D_{\text {clad }}}{d_{\text {core }}}\right)^{2}\left(\frac{\lambda_{p}}{\lambda_{s 1}}\right)<8
$$

where $D_{\text {clad }}$ and $d_{\text {core }}$ are the cladding and core diameter respectively.

Equation (4) is a basic rule of thumb for the area ratio required to avoid the $2^{\text {nd }}$ Stokes, when losses are low. Importantly, this limits the brightness enhancement that is possible. On the one hand, for a single-mode core, the maximum diameter 
is $d=2.405 \frac{\lambda}{\pi . N A}$ with NA $\sim 0.08$. On the other hand, the limited NA of the cladding means that a certain pump brightness is required. Still, it is possible to relax this condition if the loss at the $2^{\text {nd }}$ Stokes is increased. This would effectively prevent the $2^{\text {nd }}$ Stokes from building up. At the same time, to reach a high efficiency, the loss for the $1^{\text {st }}$ Stokes and the pump must remain low

\section{EXPERIMENT}

\subsection{Double-clad Raman fibre}

The double-clad Raman laser used in our experiment comprises an all-glass fibre with a silica outer cladding, a circular $20 \mu \mathrm{m}$ diameter germanium-doped inner cladding and a $9 \mu \mathrm{m}$ diameter core, which is also germanium-doped. Thus, the cladding/core area ratio is 4.9 , just within the design rule given by Eq. (4). The higher germanium concentration in the core section enhances the Raman gain there and increases the discrimination between core and cladding modes. The numerical aperture of the inner cladding is 0.22 with respect to the outer cladding and 0.14 for the core relative to the inner cladding. The measured fibre refractive index profile is shown in figure 1 . The cut-off wavelength of the core is estimated to $1640 \mathrm{~nm}$. This fibre was fabricated at the Optoelectronic Research Centre and designed originally for $1550 / 1660 \mathrm{~nm}$ operation.

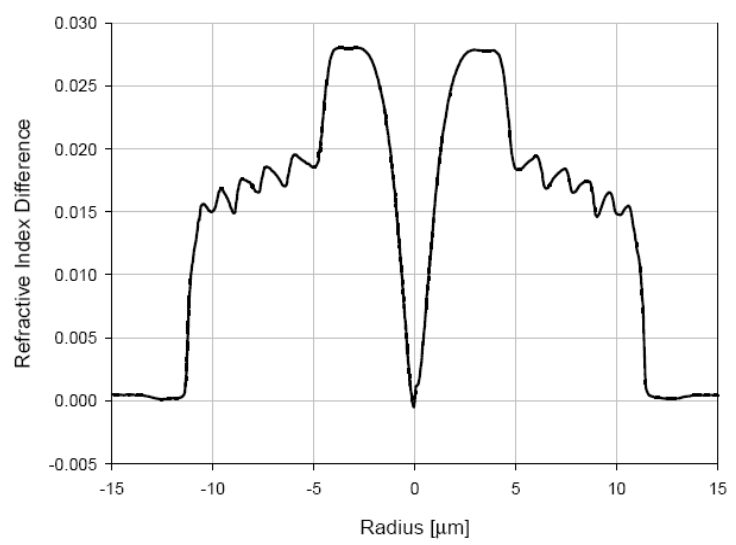

Fig. 1. Measured fibre refractive index profile

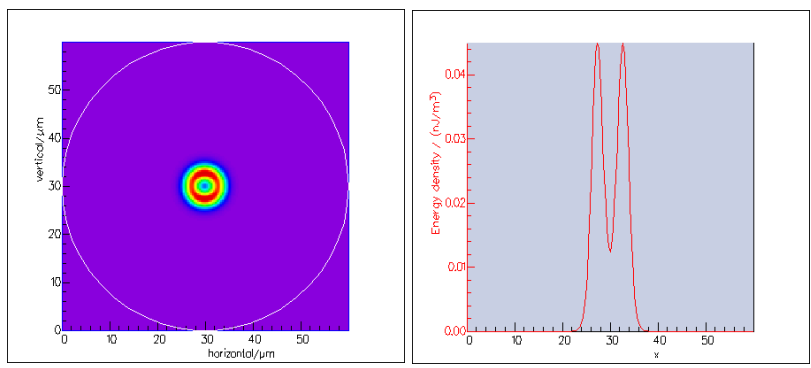

(a)

(b)

Fig. 2. Calculated $\mathrm{LP}_{01}$ mode at $1120 \mathrm{~nm}$, (a) top view, (b) transverse cross-section

Thus at the Stokes wavelength of $1112 \mathrm{~nm}$, the core is multimode with $3-5$ modes. The profile of the calculated $\mathrm{LP}_{01}$ mode is shown in figure 2. We calculated the effective area of the $\mathrm{LP}_{01}$ mode to $74.1 \mu \mathrm{m}^{2}$ and the beam propagation factor $\left(\mathrm{M}^{2}\right)$ to 1.37 at the Stokes wavelength.

\subsection{Set-up}

The experimental set-up is shown in figure 3. It is composed of three sections: a single-mode pulsed pump source at $1061 \mathrm{~nm}$, a section of multimode fibre to scramble the pump beam and thus degrade its beam quality, and the doubleclad fibre Raman laser. 


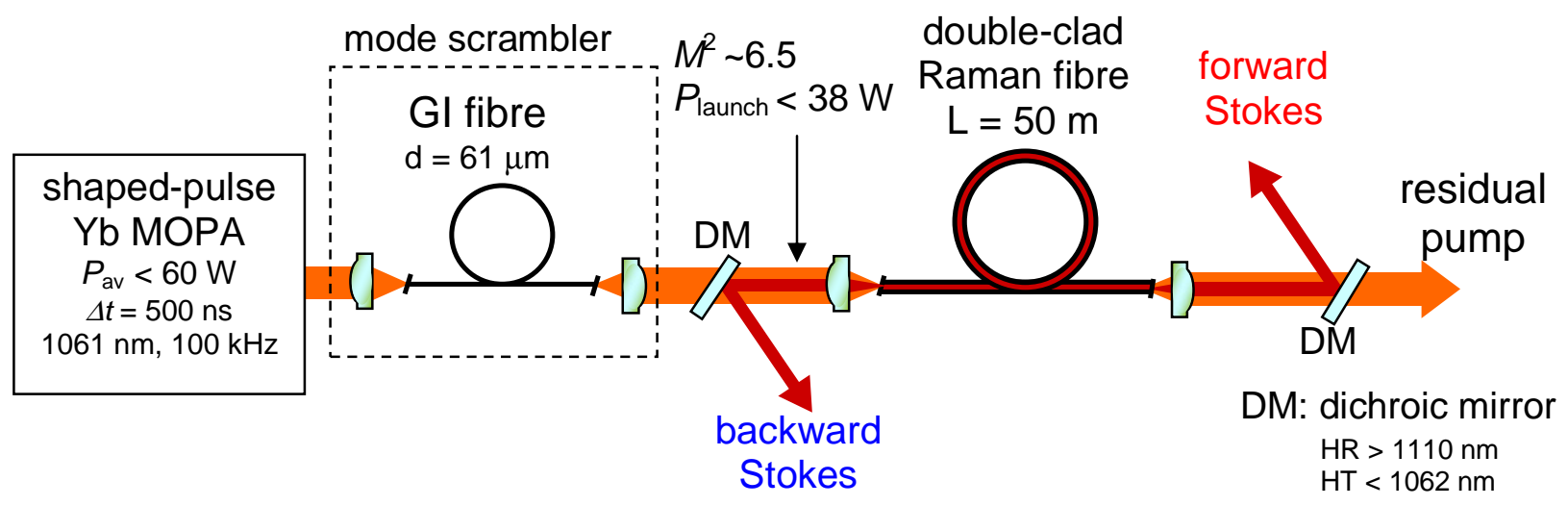

Fig. 3: Experimental set-up of the cladding-pumped fibre Raman laser

The single-mode pump source has a master-oscillator power-amplifier (MOPA) architecture and has been described in details in $[13,14]$. The pump source delivers $500 \mathrm{~ns}$ long rectangular-shaped pulses at $100 \mathrm{kHz}$ repetition rate. The average power is up to $60 \mathrm{~W}$ and the wavelength is $1061 \mathrm{~nm}$. Pre-shaping of the master oscillator allows us to obtain rectangular pulses which are desirable for efficient Raman conversion [14]. Then the pump light is free-space launched into a $1-2 \mathrm{~m}$ long piece of graded-index (GI) multimode fibre to degrade the beam quality without generating any Raman scattering. The beam propagation factor is measured to be 6.5 at the output of the GI fibre. This multimode light beam is then free-space launched into a $50 \mathrm{~m}$ long piece of the DCRF via dichroic mirrors (DM) and lenses. The launch efficiency into the Raman fibre is $\sim 75 \%$. From the fibre details given above and the pulse duration, the pulse walk-off

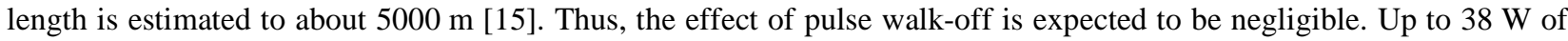
pump average power is launched into the DCRF. We used dichroic mirrors with sharp discrimination to separate the Stokes and pump wavelengths in the forward and backward propagation directions. In addition, the front-end dichroic mirror suppresses any Raman-scattered light generated in the pump source. The Raman Stokes beam builds up from spontaneous Raman scattering in the DCRF, since there is no separate Raman seed source. 


\section{RESULTS}

\subsection{Output Power and Energy}

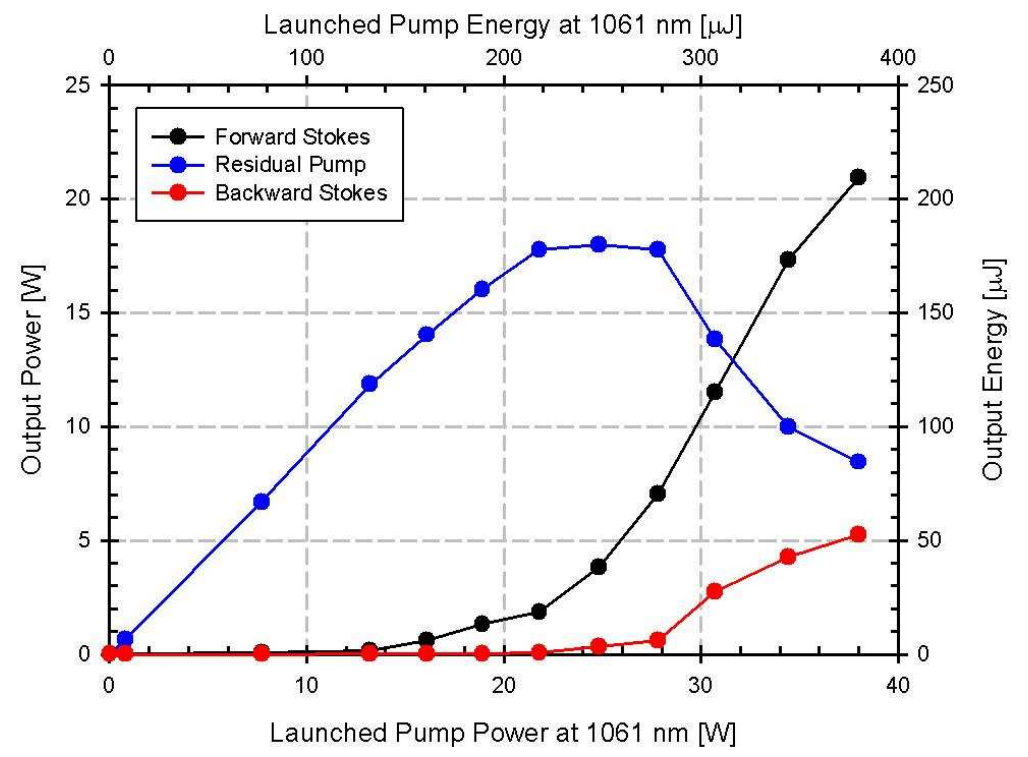

Fig. 4: Output average power and output energy with respect to launched pump power and energy for the forward and backward Stokes and residual pump.

The average output power and the output energy of the forward and backward Stokes as well as remaining pump power are shown in figure 4. At the maximum launched pump power of $38 \mathrm{~W}$ (average power), the forward Stokes power reaches $21 \mathrm{~W}$. This corresponds to an $210 \mu \mathrm{J}$ Stokes pulse with a peak power of $420 \mathrm{~W}$. The Stokes intensity in the core is $1-2.8 \mu \mathrm{J} / \mu^{2}$ just below the damage threshold [16] for $500 \mathrm{~ns}$ long pulses. Here the core size limits the power scaling of this fibre with these pump pulses.

The energy conversion efficiency with respect to launched energy is 55\% into the forward Stokes. This is partly limited by the generation of backward Stokes light. The overall pump to Stokes conversion efficiency is $88 \%$ with respected to absorbed pump power.

\subsection{Spectra}

The forward output spectra are shown in figure 5 for various pump power levels. At the maximum pump energy of $380 \mu \mathrm{J}$, some $2^{\text {nd }}$ Stokes light is generated. A detail analysis shows that the energy in the $1^{\text {st }}$ Stokes is $188 \mu \mathrm{J}$ out of $210 \mu \mathrm{J}$ in the forward Stokes. The 3 linewidth of the $1^{\text {st }}$ Stokes at $1112 \mathrm{~nm}$ is $6.5 \mathrm{~nm}$ (full width at half maximum). 


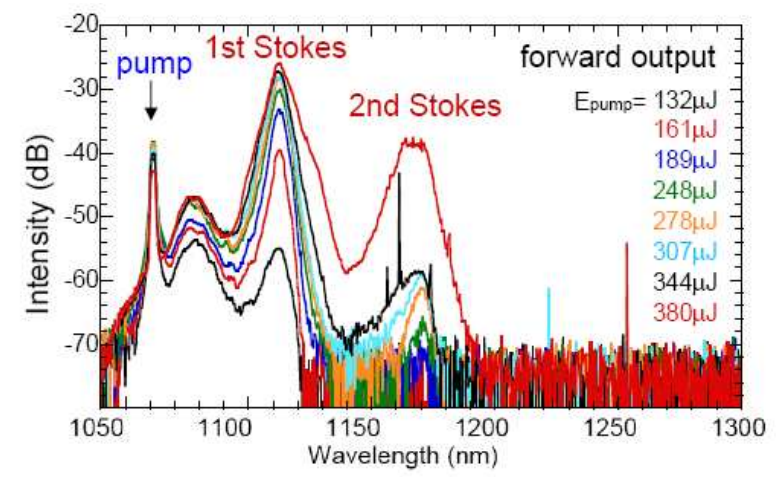

Fig. 5: Forward output spectra for different pump energy levels. Optical resolution $2 \mathrm{~nm}$.

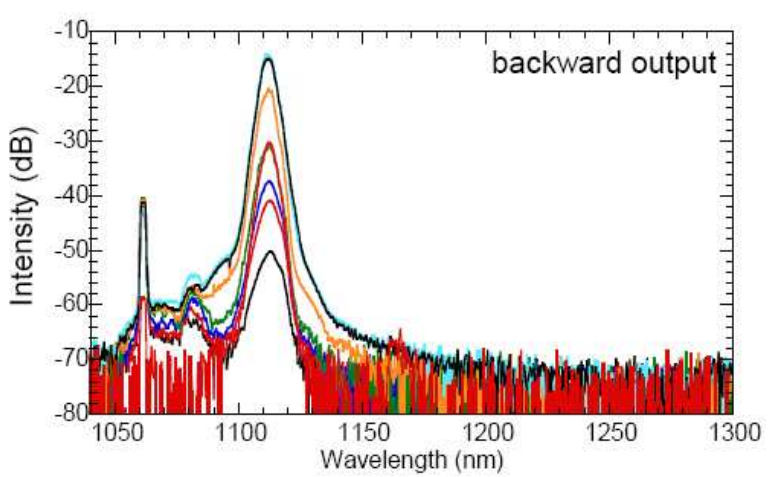

Fig. 6: Backward output spectra for the same energy level as fig. 5. Optical resolution $2 \mathrm{~nm}$.

The spectra of the backward light are shown in figure 6 . Here only the light at the $1^{\text {st }}$ Stokes can be seen. This is explained by the shorter interaction length of the backward Stokes as detailed below in our temporal pulse analysis.

\subsection{Beam quality}

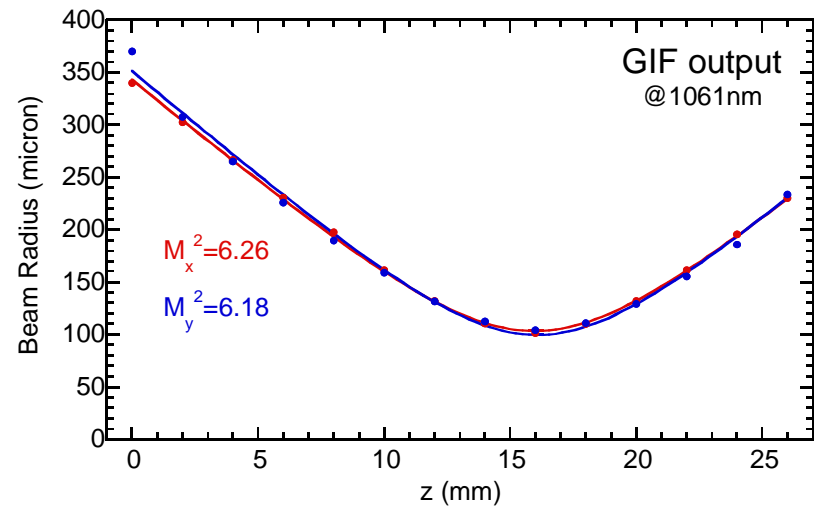

Fig. 7: Beam propagation measurement of the input forward pump light after the graded-index fibre (GIF).

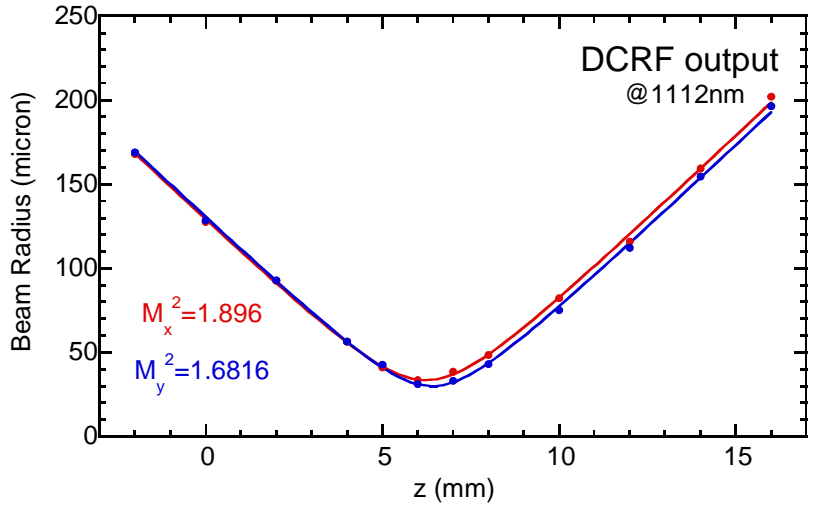

Fig. 8: Beam propagation measurement of the output forward Stokes light from the DCRF.

The beam propagation factor $\left(\mathrm{M}^{2}\right)$ measurement of the input and DCRF output light beam are shown in figure 7 and figure 8, respectively. The average $\mathrm{M}^{2}$ is 6.22 for the pump at the maximum pump power and for the forward Stokes the $\mathrm{M}^{2}$ is 1.79 . Although the output of the DCRF is not diffraction-limited, it is not far from the calculated $\mathrm{M}^{2}$ of 1.37 for the $\mathrm{LP}_{01}$ mode. Using the definition of the brightness enhancement factor [15] given by:

$$
\eta_{B}=\frac{B_{\text {Stokes }}}{B_{\text {pump }}}=\frac{E_{\text {Stokes }}}{E_{\text {pump }}}\left(\frac{M_{\text {pump }}^{2} \lambda_{\text {pump }}}{M_{\text {Stokes }}^{2} \lambda_{\text {Stokes }}}\right)^{2}
$$

the conversion of the $\mathrm{M}^{2}$ of the pump into the Stokes represents a brightness enhancement of $\sim 6$. Thus, the Ramanconverted output beam is 6 times brighter than the pump beam just by using a passive piece of fibre. 


\subsection{Temporal pulse profiles}

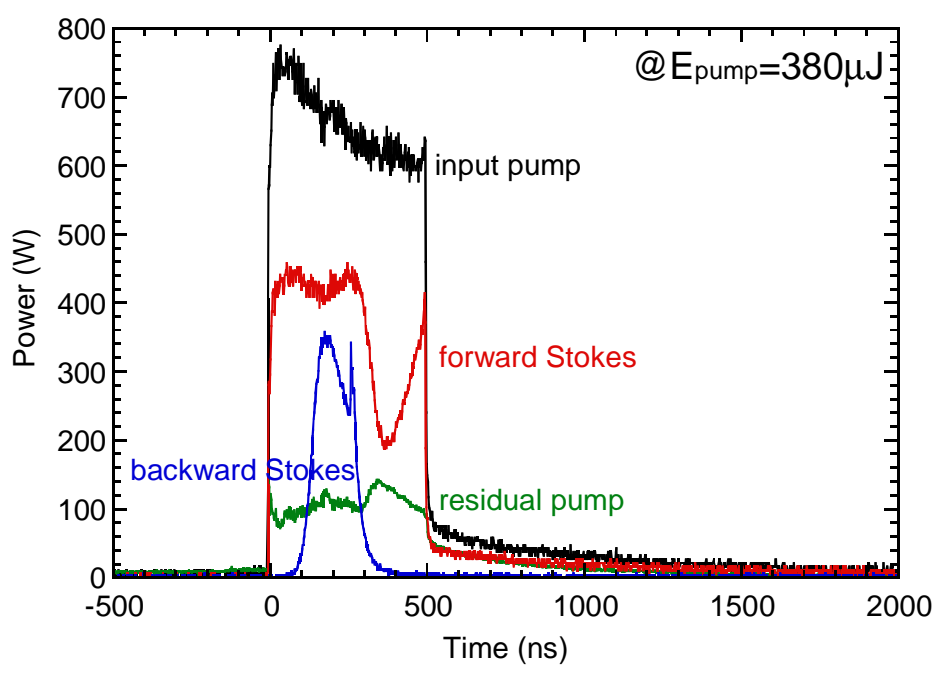

Fig. 9: Temporal pulse profile at the maximum launched pump power of the input pump, forward and backward Stokes and the residual pump.

The temporal pulse profiles for the forward and backward Stokes, as well as launched and remaining pump, are shown in Fig. 9. For a $380 \mu \mathrm{J}$ pump pulse, the residual pump energy is $84 \mu \mathrm{J}$ while the forward and backward Stokes energy are 210 and $53 \mu \mathrm{J}$, respectively. Clearly, the pump power is not completely depleted. We believe that some pump light might have a weaker overlap with the Stokes modes. The circular shape of the cladding is not optimum for claddingpumping [17] but we are restricted by the current fabrication method of the all-glass structure.

Note that the forward Stokes profile has a dip in its trailing end. This is due to depletion of the forward-propagating pump induced by the backward-propagating Stokes beam. Our pulses are significantly longer than the fibre; in fact more than twice as long ( $50 \mathrm{~m} \leftrightarrow 242 \mathrm{~ns}$ ). Thus any scattering from the leading edge of a pulse is amplified by the latter part of the pump pulse, and the trailing edge of the pump pulse is depleted by backward-propagating Stokes light that has been fully amplified first in the forward and then in the backward direction through the fibre. As a result we observe a depletion of the trailing edge of the forward Stokes pulse, and significant power is lost in the backward direction. This effect can be mitigated by using shorted pulse durations with the same peak power. The energy then needs to be decreased as the launched pump intensity is limited to approximately $10-20$ times $\left(L g_{R}\right)^{-1}$ or $4-8 \mathrm{~W} / \mu \mathrm{m}^{2}$ for our fibre, if higher-order Raman scattering is to be avoided. Here, $L$ is the fibre length and $g_{R}$ is the Raman gain coefficient. This limits the pump pulse energy fluence to $1-2 \mu \mathrm{J} / \mu \mathrm{m}^{2}$, for a well-designed Raman fibre, if the pulses are to be shorter than the fibre. (Note that since we used longer pulses this limitation does not strictly apply in our case.)

\section{CONCLUSION}

In this work, we have presented design requirements for a cladding-pumped Raman fibre and have experimentally shown a high-energy pulsed cladding-pumped Raman fibre laser. Stokes pulses in the forward direction reached output energies of $210 \mu \mathrm{J}$ at $420 \mathrm{~W}$ of average power. This corresponds to a brightness enhancement factor of 6.1. In this Raman laser, in which the emission builds up from spontaneous Raman scattering, the backward Stokes generation limits efficiency and energy in the forward direction. This can be mitigated by adjusting the pump peak power and duration with respect to the fibre length. Still, the construction of this brightness-enhancer is simple and it can be added to any multimode pump source depending on the pump power and pump brightness. The overall energy conversion efficiency with respect to absorbed pump power is $88 \%$. We believe that this method is an elegant solution for creating 
high-brightness fibre laser sources and that it is capable of further power and energy scaling with diffraction-limited output and even higher conversion efficiency.

This work was financed by EPSRC.

\section{REFERENCES}

[1] information available from IPG Photonics: http://www.ipgphotonics.com.

[2] Y. Jeong, et al, "120-W Q-switched cladding-pumped Yb-doped fibre laser", in Conf. Lasers and Electro-Optics Eur., 27E, Europhysics Conf. Abstracts, 2003, Paper CL5-4.

[3] J. Nilsson, et al., "Cladding-pumped Raman amplifier", in OAA (2002) Vancouver, Canada 14-17 Jul 2002 PD2.

[4] S. H. Baek, and W. B. Roh, "Single-mode Raman fiber laser based on a multimode fiber", Opt. Lett. 29, 153-155 (2004).

[5] C. A. Codemard, et al., "High-power continuous-wave cladding-pumped Raman fiber laser", Opt. Lett. 31, 2290$2292(2006)$.

[6] J. N. Jang, et al., "Cladding-pumped continuous-wave Raman fiber laser", in Conf. Lasers and Electro-Optics/QELS 2003 Baltimore 3-5 Jun 2003.

[7] C. A. Codemard, et al., "Cladding-pumped Raman fiber amplifier for high-gain high-energy single-stage amplification", in OFC (2005), 6-11 March 2005, Anaheim, CA, USA.

[8] C. A. Codemard, et al., "High-brightness pulsed cladding-pumped Raman fibre source at $1660 \mathrm{~nm}$ ", in Conf. Lasers and Electro-Optics /QELS 2007 Baltimore 6-11 May 2007 CTuN3

[9] R. S. F. Chang,, et al., "Raman Beam Cleanup of a Severely Aberrated Pump Laser", IEEE J. Quantum Electron. 21(5), 477-487 (1985).

[10] N. B. Terry, T. G. Alley, and T. H. Russell, "An explanation of SRS beam cleanup in graded-index fibers and the absence of SRS beam cleanup in step-index fibers," Opt. Express 15, 17509-17519 (2007)

[11] N. B. Terry, et al., "Use of a continuous wave Raman fiber laser in graded-index multimode fiber for SRS beam combination", Opt. Express 15, 602-607 (2007).

[12] G. P. Agrawal, Nonlinear Fiber Optics, (2 ${ }^{\text {nd }}$ Ed., Academic Press Inc, San Diego CA, 1995).

[13] K .T. Vu, et al., "Adaptive pulse shape control in a diode-seeded nanosecond fiber MOPA system", Opt. Express 14, 10996-11001 (2006).

[14] A. Malinowski,, et al., "Selective generation of individual Raman Stokes wavelengths using shaped optical pulses", in OFC 2008 San Diego 24-28 Feb 2008 OTuB3

[15] C. A. Codemard, PhD Thesis, University of Southampton, "High-power cladding-pumped Raman and erbiumytterbium doped fibre sources" (2007).

[16] W. Köchner: Solid-State Laser Engineering (Springer Series in Optical Science, Berlin 1999)

[17] A. Liu, K. Ueda, "The absorption characteristics of circular, offset, and rectangular double-clad fibers", Opt. Commun. 132(5-6) 511 (1996). 